\title{
Efficacy of a novel herbal composition licorice flavonoid oil in subject with metabolic syndrome: a randomized double-blind placebo-controlled clinical study
}

\section{Sanjib Kumar Panda ${ }^{1}$, Kaku Nakagawa ${ }^{2}$, Takahiro Ueda ${ }^{2}$, Masato Hosoda ${ }^{2}$, Kazunori Hosoe $^{2}$, Hideyuki Morikawa ${ }^{2}$ and Sandeep $\mathrm{Jha}^{3}$}

${ }^{1}$ Ocius Life Sciences Private Ltd., 325, P.H.Road, Aminjikarai, Chennai - 600029, India; ${ }^{2}$ QOL Division, Kaneka Corporation, 2-3-18 Nakanoshima, Kita-Ku, Osaka 530-8288, Japan; ${ }^{3}$ Generix Life Sciences Private Ltd., 903, Tower A-1, Ansal Corporate Park, Plot No 7A/1, Noida Expressway, Behind Advant Building, Sector 142, Noida - 201301, India

Corresponding author: Kazunori Hosoe, $\mathrm{PhD}$, QOL Division, Kaneka Corporation, 2-3-18 Nakanoshima, Kita-Ku, Osaka, 530-8288, Japan

Submission Date: January $30^{\text {th }}$, 2017, Acceptance date: March 28 ${ }^{\text {th, }}$ 2017: Publication date: March 31, 2017

Citation: Panda S.K., Nakagawa K., Ueda T., Hosoda M., Hosoe K., Morikawa H., Jha S. Efficacy of a novel herbal composition licorice flavonoid oil in subject with metabolic syndrome: a randomized double-blind placebo-controlled clinical study. Functional Foods in Health and Disease 2017; 7(3): 210-221

\begin{abstract}
Background: In order to evaluate the effects of licorice flavonoid oil (LFO) on abdominal waist circumference, blood pressure, body weight, body mass index (BMI), lipid profile, body fat composition, and fasting blood glucose in patients with metabolic syndrome, a 12 week randomized double-blind placebo-controlled study was conducted.
\end{abstract}

Methods: Fifty patients with metabolic syndrome aged between 18-75 years were assigned to either the LFO or placebo group. Abdominal waist circumference, blood pressure, body weight, and BMI were assessed at baseline, week 4, week 8, and week 12. Clinical laboratory examinations, fat composition, and fasting blood glucose level were assessed at baseline (week 0) and final visit (week 12).

Results: A total of 50 subjects (25 subjects in each group) completed the 12-week study. Significant difference in changes from the baseline was observed in body weight, waist 
circumference, and BMI in the LFO group compared to the placebo group from week 4 or week 8 onwards. No adverse events were noted throughout the study.

Conclusion: The present study suggests that LFO is a promising dietary nutrient for improving metabolic syndrome, particularly through its beneficial effect of normalizing body weight, BMI, and possibly the amount of visceral fat and HDL cholesterol.

Keywords: licorice flavonoid oil; Glycyrrhiza glabra L.; metabolic syndrome; body mass index

\section{BACKGROUND}

The metabolic syndrome has garnered great attention since its definition was introduced globally by the World Health Organization in 1999 as a serious risk factor for life-threatening diseases, including type 2 diabetes and cardiovascular diseases [1,2]. Food supplements or nutraceuticals including antioxidants, dietary fiber, and omega-3 polyunsaturated fatty acid-containing lipids, in combination with lifestyle modifications, have been clinically shown to be beneficial in preventing or alleviating metabolic syndrome [3-5].

We have also discovered a new and safe dietary ingredient of licorice flavonoid oil (LFO), consisting of hydrophobic polyphenols derived from licorice (Glycyrrhiza glabra L.) and dissolved in medium-chain triglycerides (MCT) is a beneficial candidate for treating metabolic syndrome; this conclusion is based on the fact that glabridin, a major isoflavan in Glycyrrhiza glabra L., is one of the well-defined flavonoids with anti-oxidative [6-10], anti-H. pylori [11], in addition to anti-nephritic and radical scavenging activities [12]. This substance was also reported to inhibit serotonin re-uptake [13], melanogenesis and inflammation [14].

The safety of LFO has been confirmed in animals as well as within humans [15-19] and while effects, including the suppression of weight gain and blood glucose elevation, and reduction of the accumulation of visceral fat, were demonstrated in obese diabetic KK-Ay mice [20].

Accordingly, randomized, double-blind, placebo-controlled studies conducted with moderately overweight healthy subjects have suggested that the ingestion of 3-fold diluted LFO at a daily dose of $900 \mathrm{mg}$ (equivalent to $300 \mathrm{mg}$ of Flabonoid ${ }^{\mathrm{TM}}$, which was used in the present study) is effective in suppressing body weight gain and BMI increase [19] or in reducing body weight, body mass index (BMI), total body fat mass, visceral fat area, and LDL-cholesterol, with no significant adverse effects [21].

In the present study, for the first time we assessed the efficacy of Flabonoid ${ }^{\mathrm{TM}}$ in subjects with metabolic syndrome at a daily dosage of $300 \mathrm{mg} /$ day for 12 weeks, while also evaluating its effects on body weight, BMI, waist circumference, blood lipid profile, fasting blood glucose level, blood pressure, and body composition. 


\section{METHODS}

\section{Subjects}

Fifty Indian patients between the ages of 18 and 75 who were diagnosed with metabolic syndrome according to criteria of the 2005 revised National Cholesterol Education Program Adult Treatment panel III (revised NCEP ATP III) criteria [22] from a single site were enrolled. The revised NCEP ATP III requires at least three or more of the following components: (1) abdominal obesity (waist circumference $>90 \mathrm{~cm}$ for Asian men or $>80 \mathrm{~cm}$ for Asian women), (2) triglycerides $\geq 150 \mathrm{mg} / \mathrm{dL}$, (3) HDL cholesterol $<40 \mathrm{mg} / \mathrm{dL}$ for men or $<50 \mathrm{mg} / \mathrm{dL}$ for women, (4) systolic/diastolic blood pressure $\geq 130 / 85 \mathrm{mmHg}$ or receiving drug treatment, and (5) fasting plasma glucose $\geq 100 \mathrm{mg} / \mathrm{dL}$. Exclusion criteria for participation included pregnancy or breast-feeding; uncontrolled hypertension, diabetes, other cardiovascular diseases, or previous cardiovascular events including angina pectoris, heart failure, and myocardial infraction; abnormal liver or kidney function (ALT or AST $>2$ times the upper limit of normal); elevated creatinine (males $>125 \mu \mathrm{mol} / \mathrm{L}$, females $>110 \mu \mathrm{mol} / \mathrm{mL}$ ); renal insufficiency; thyroid or other endocrine diseases; currently undergoing treatment for cancer or taking medications that are known to modify signs and other parameters of metabolic syndrome. For this first pilot study with LFO in subjects with metabolic syndrome, the sample size of 50 was determined based on a previous study in healthy subjects conducted to evaluate effect of LFO on body weight, body fat, and visceral fat [21], while also following general suggestions by Julious SA (2005) [23] and Hertzog MA (2008) [24]. The patients were randomly assigned with the use of a computer-generated, permuted-block design. The nature and risks of the study medication were explained to the subjects before the informed consent form was obtained. The first subject for this study was recruited on Oct $30^{\text {th }}, 2015$ and the last subject completed their last visit on Feb $15^{\text {th }}, 2016$.

\section{Study design and data collection}

This randomized, double-blind, parallel placebo-controlled study was reviewed and approved by the Institutional Review Board (IRB) of Rajiv Gandhi Institute of Medical Sciences \& RIMS Govt. General Hospital, India and was conducted in accordance with the requirements of the Declaration of Helsinki in its latest form and current ICH-GCP (International Conference on Harmonization-Good Clinical Practice) standards. Subjects were instructed to take one capsule once a day with meals for 12 weeks. The subjects were required to make five visits to the clinical site during the study tenure as follows: at the screening visit, at baseline, week 4 (day 28), week 8 (day 56) and week 12 (day 84; end of the study). At each visit, body weight, BMI, waist circumference, blood pressure, and study compliance were assessed. Clinical Laboratory parameters and body composition (bioelectrical impedance analyzer DC-320, Tanita, Corp., Tokyo, Japan) were examined at baseline (week 0) and final visit (week 12). The primary endpoints were changes in waist circumference, triglycerides and HDL. 


\section{Test food supplement}

To prepare the LFO test material (Flabonoid ${ }^{\mathrm{TM}}$, Generix Life Sciences Private Ltd), an ethanol extract of Glycyrrhiza glabra L. was mixed with MCT and the concentration of glabridin, which is the main component of flavonoids, was adjusted to $3 \%(\mathrm{w} / \mathrm{w})$. Then, soft gelatin capsules containing $300 \mathrm{mg}$ of Flabonoid ${ }^{\mathrm{TM}}$ per capsule were then prepared with MCT and beeswax. Matching placebo capsules were also prepared.

\section{Clinical laboratory examinations}

Hematology (hemoglobin, platelet count, total leukocytes, differential count), fasting blood glucose, SGOT, SGPT, HbA1C, and serum lipid profile (LDL cholesterol, HDL cholesterol, triglycerides, total cholesterol) were determined at the baseline (week 0) and final visit (week 12).

\section{Statistical analyses}

Data are presented as mean \pm S.D. Within-group differences between baseline and post-intervention data were analyzed by Wilcoxon signed-rank test, while between-group differences were assessed with Mann-Whitney's U test. For frequency data, chi-square test was used. Analyses were performed with SAS preclinical package software, version 9.4 (SAS system; SAS Institute Japan Ltd., Tokyo, Japan). P value of $<0.05$ was considered to be statistically significant.

\section{RESULTS}

A total of 50 subjects with metabolic syndrome were randomized to two groups of 25 subjects. All enrolled subjects completed the study. As shown in Table 1, there was no significant difference was seen in age, body height, weight, BMI and male ratio between both groups at the baseline visit. There were no adverse events reported in both groups during the study tenure.

Table 1. Subject characteristics at baseline

\begin{tabular}{lccc}
\hline & LFO & Placebo & P value $^{\#}$ \\
\hline Number of subjects & 25 & 25 & - \\
Age (years) & $33.8 \pm 9.5$ & $35.2 \pm 9.8$ & 0.540 \\
Number of males & 19 & 17 & 0.529 \\
Body weight $(\mathrm{kg})$ & $73.9 \pm 9.7$ & $73.9 \pm 12.0$ & 0.793 \\
Height $(\mathrm{cm})$ & $161.3 \pm 8.1$ & $160.1 \pm 6.0$ & 0.566 \\
BMI $\left(\mathrm{kg} / \mathrm{m}^{2}\right)$ & $28.4 \pm 2.7$ & $28.8 \pm 3.7$ & 0.786 \\
\hline
\end{tabular}

Data are expressed as mean \pm SD except for number of males.

${ }^{\#} \mathrm{P}$ value for Chi-Square test for number of males or Mann-Whitney's U test for other data. 
Additionally, no significant changes in laboratory parameters and vital signs were noted when compared to the baseline, except the results described in the following paragraphs and Table 2 and Table 3, all of which contained noteworthy observations. Mean changes from baseline to 12 weeks in body weight, waist circumference and BMI is shown in Figure 1.
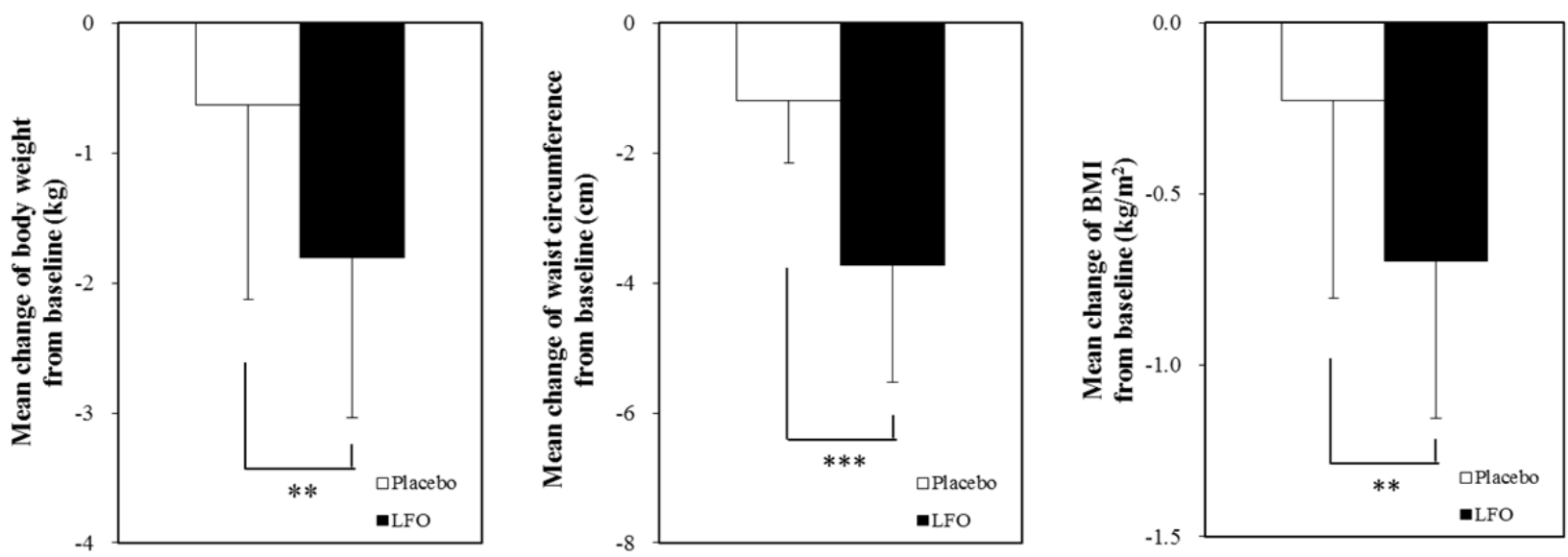

Figure 1. Mean changes from baseline to 12 weeks in body weight, waist circumference, and BMI. Data are expressed as mean $\pm \mathrm{SD}$. ${ }^{* *} \mathrm{p}<0.01,{ }^{* * *} \mathrm{p}<0.001$ by Mann-Whitney's U test.

During the study period, a significant reduction in body weight from baseline was observed at week $4(\mathrm{p}<0.01), 8(\mathrm{p}<0.001)$ and $12(\mathrm{p}<0.001)$ in the LFO group and at week $4(\mathrm{p}<0.05)$ and 12 $(\mathrm{p}<0.05)$ in the placebo group. However, the degree of reduction in the LFO group at week 8 $(\mathrm{p}<0.001)$ and $12(\mathrm{p}<0.01)$ was significantly greater than in the placebo group (Table 2$)$.

A significant reduction in waist circumference from baseline was also observed in both groups $(\mathrm{p}<0.05, \mathrm{p}<0.001, \mathrm{p}<0.001$ at week 4,8 , and 12 respectively in the LFO group and $\mathrm{p}<0.001$ in all visits in the placebo group). The mean reduction in waist circumference at week 4, 8, and 12 was $0.4,1.7$, and $3.7 \mathrm{~cm}$ respectively for the LFO group, and $0.8,0.8$, and $1.2 \mathrm{~cm}$, respectively for the placebo group. The reduction at week 8 and 12 in the LFO group was statistically greater than in the placebo group $(\mathrm{p}<0.01$ and $\mathrm{p}<0.001$, respectively) (Table 2 ).

Similar to the observations on body weight and waist circumference, a significant reduction in BMI from baseline was observed in both groups $(\mathrm{p}<0.01, \mathrm{p}<0.001, \mathrm{p}<0.001$ at week 4, 8, and 12 respectively in the LFO group, in addition to $\mathrm{p}<0.05$ at week 4 and 12 in the placebo group). However, the mean reduction in BMI at week 4,8 , and 12 was $0.3,0.8$, and $0.7 \mathrm{~kg} / \mathrm{m}^{2}$ respectively for the LFO group, and $0.3,0.1$, and $0.2 \mathrm{~kg} / \mathrm{m}^{2}$ respectively for the placebo group. The degree of reduction in the LFO group at week $8(\mathrm{p}<0.001)$ and $12(\mathrm{p}<0.01)$ was significantly greater than in the placebo group (Table 2).

As for systolic blood pressure, there were no significant changes from the baseline for both groups, except at week 12 in LFO group, and no significant differences between the LFO group and the placebo group were observed at all time points. While a significant increase in systolic blood pressure was observed in LFO group $(\mathrm{p}<0.05)$ at week 12 , the physiological significance of 
this is considered minimal as the observed changes of $4.2 \mathrm{mmHg}$ from the baseline was small, with the absolute values also being within normal ranges. As for diastolic blood pressure, a significant increase from baseline was observed only in the placebo group at week 4 and 8 ( $p<0.01$ for both time points), but not at week 12. A significant difference between groups was observed at week 4 and 8 ( $\mathrm{p}<0.05$ for both time points), with the physiological significance of this also being considered minimal as the observed changes of 2.1 and $2.4 \mathrm{mmHg}$ at week 4 and 8 respectively from the baseline level were small, and the absolute values in the placebo group were still within the reference ranges (Table 2).

Table 2. Changes in body weight, waist circumference, BMI, systolic blood pressure, and diastolic blood pressure

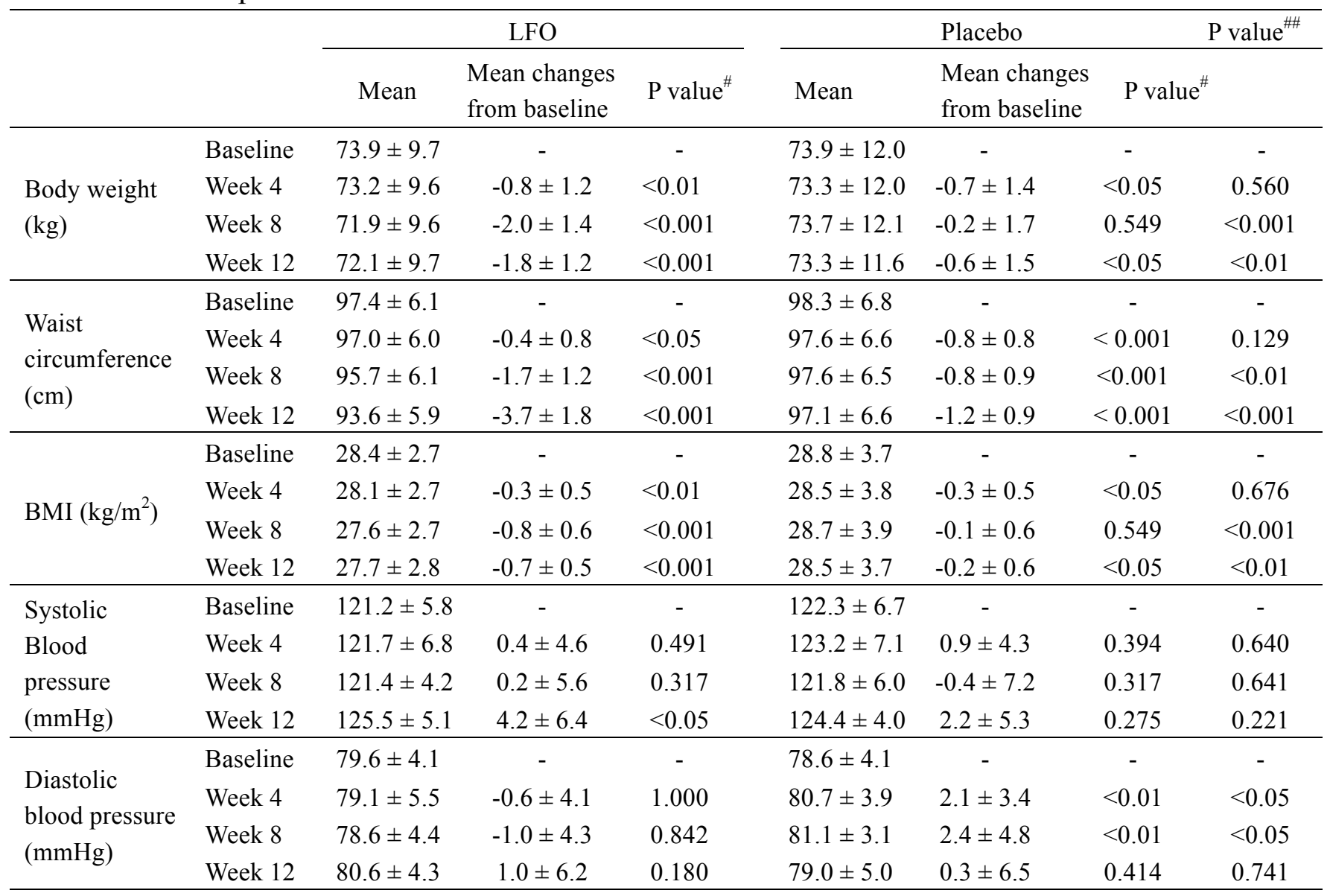

Data are expressed as mean $\pm \mathrm{SD}$.

${ }^{\#} \mathrm{p}$ value for Wilcoxon signed-rank test (within-group differences in mean values).

${ }^{\#}$ p value for Mann-Whitney's U test (between-group differences in mean changes from baseline).

Regarding the assessment of the lipid profile and glycemic control, significant changes were seen in triglycerides $(\mathrm{p}<0.001)$ along with a significant increase in HDL cholesterol values $(\mathrm{p}<0.05$ or $\mathrm{p}<0.01)$ from the baseline for both groups. However, there was no significant difference in these parameters noted between the two groups, although HDL cholesterol values in 
LFO group demonstrated a tendency $(\mathrm{p}=0.075)$ to be greater than that in placebo group. Additionally, no significant change in LDL values in the LFO group was observed and the placebo group demonstrated a significant decrease in LDL cholesterol from baseline $(\mathrm{p}<0.05)$. However, there was no significant difference between the two groups with regards to the decrease in LDL cholesterol values. There was also no significant decrease from baseline in total cholesterol values in both groups and no significant difference in the changes from baseline between the two groups (Table 3).

Table 3. Changes in serum lipid profile, fating blood glucose, glycosylated hemoglobin, and body fat composition

\begin{tabular}{|c|c|c|c|c|c|c|c|c|}
\hline & & \multicolumn{3}{|c|}{ LFO } & \multicolumn{3}{|c|}{ Placebo } & \multirow[t]{2}{*}{ P value ${ }^{\# \#}$} \\
\hline & & Mean & $\begin{array}{l}\text { Mean changes } \\
\text { from baseline }\end{array}$ & P value ${ }^{\#}$ & Mean & $\begin{array}{l}\text { Mean changes } \\
\text { from baseline }\end{array}$ & P value ${ }^{\#}$ & \\
\hline \multirow{2}{*}{$\begin{array}{l}\text { Triglycerides } \\
(\mathrm{mg} / \mathrm{dL})\end{array}$} & Baseline & $175.7 \pm 13.0$ & - & - & $181.8 \pm 9.1$ & - & - & - \\
\hline & Week 12 & $160.7 \pm 9.8$ & $-15.0 \pm 9.5$ & $<0.001$ & $170.9 \pm 11.7$ & $-11.0 \pm 11.5$ & $<0.001$ & 0.316 \\
\hline \multirow{2}{*}{$\begin{array}{l}\text { HDL } \\
(\mathrm{mg} / \mathrm{dL})\end{array}$} & Baseline & $36.9 \pm 3.1$ & - & - & $36.8 \pm 3.6$ & - & - & - \\
\hline & Week 12 & $42.5 \pm 7.0$ & $5.6 \pm 6.1$ & $<0.01$ & $39.7 \pm 4.9$ & $2.9 \pm 4.6$ & $<0.05$ & 0.075 \\
\hline \multirow{2}{*}{$\begin{array}{l}\mathrm{LDL} \\
(\mathrm{mg} / \mathrm{dL})\end{array}$} & Baseline & $166.4 \pm 8.7$ & - & - & $171.9 \pm 11.3$ & - & - & - \\
\hline & Week 12 & $160.6 \pm 15.2$ & $-5.7 \pm 15.3$ & 0.317 & $165.1 \pm 13.9$ & $-6.8 \pm 10.3$ & $<0.05$ & 0.923 \\
\hline \multirow{2}{*}{$\begin{array}{l}\text { Total } \\
\text { Cholesterol } \\
(\mathrm{mg} / \mathrm{dL}) \\
\end{array}$} & Baseline & $191.4 \pm 17.0$ & - & - & $191.9 \pm 16.7$ & - & - & - \\
\hline & Week 12 & $185.8 \pm 18.9$ & $-5.6 \pm 16.9$ & 0.162 & $188.1 \pm 15.8$ & $-3.8 \pm 16.8$ & 0.317 & 0.648 \\
\hline \multirow{2}{*}{$\begin{array}{l}\text { Fasting blood } \\
\text { glucose } \\
(\mathrm{mg} / \mathrm{dL})\end{array}$} & Baseline & $102.0 \pm 7.5$ & - & - & $104.4 \pm 10.3$ & - & - & - \\
\hline & Week 12 & $100.0 \pm 7.9$ & $-2.0 \pm 9.4$ & 0.549 & $99.1 \pm 10.2$ & $-5.3 \pm 11.4$ & 0.072 & 0.156 \\
\hline \multirow[t]{2}{*}{ HbA1c (\%) } & Baseline & $5.6 \pm 0.4$ & - & - & $5.7 \pm 0.5$ & - & - & - \\
\hline & Week 12 & $5.5 \pm 0.3$ & $-0.0 \pm 0.2$ & 0.842 & $5.6 \pm 0.4$ & $-0.0 \pm 0.3$ & 0.549 & 0.882 \\
\hline \multirow{2}{*}{$\begin{array}{l}\text { Body fat } \\
\text { composition } \\
(\%)\end{array}$} & Baseline & $35.5 \pm 5.9$ & - & - & $36.2 \pm 6.4$ & - & - & - \\
\hline & Week 12 & $34.6 \pm 6.5$ & $-0.9 \pm 4.2$ & 0.549 & $36.6 \pm 6.7$ & $0.4 \pm 3.6$ & 0.549 & 0.861 \\
\hline
\end{tabular}

Data are expressed as mean $\pm \mathrm{SD}$.

\# $\mathrm{p}$ value for Wilcoxon signed-rank test (within-group differences in mean values).

${ }^{\#} \mathrm{p}$ value for Mann-Whitney's U test (between-group differences in mean changes from baseline).

Regarding glycemic control, no significant decrease from the baseline was seen in fasting blood glucose values for both group, and there were no significant differences in the changes from the baseline between the two groups. Additionally, neither group demonstrated a significant decrease in glycosylated hemoglobin values from the baseline, with no significant changes in this parameter being noted between the two groups as well (Table 3).

An analysis of body fat composition at baseline and at the final visit of week 12 showed no significant decrease from the baseline in both groups, in addition to no significant difference in changes between the two groups (Table 3). 
The number of subjects who showed normal value compared to each of the criteria of metabolic syndrome at baseline and week 12 in LFO group were 0 and 7 in waist circumference, 8 and 14 in fasting glucose, 1 and 13 in HDL cholesterol, and 0 and 1 in triglyceride respectively, while those in the placebo group were 1 and 3 in waist circumference, 7 and 12 in fasting glucose, 3 and 9 in HDL cholesterol, and 0 and 0 in triglyceride respectively. In regard to blood pressure, 24 subjects in LFO group and 25 subjects in placebo group showed normal blood pressure values at the baseline and at week 12. One subject in the LFO group with abnormal values at baseline did not show any improvement at week 12 . Therefore, it might be difficult to detect the effect of LFO on blood pressure in this study. Furthermore, the prevalence of subjects who showed improvement in both waist circumference and HDL cholesterol in LFO group at week 12 was 5 out of 25, which was significantly higher than 0 out of 25 in the placebo group $(p<0.05)$. Consequently, the percentage of subjects not categorized as having metabolic syndrome was 48 in the LFO group and 24 in the placebo group at week 12, with no significant statistical difference between the two groups. However, a tendency toward a high percentage of subjects not categorized as having metabolic syndrome was seen in the LFO group compared to the placebo group $(p=0.077)$.

\section{DISCUSSION}

In this study, we evaluated the effects of LFO at an oral daily dose of $300 \mathrm{mg}$ for 12 weeks on various indexes in subjects with metabolic syndrome. The ingestion of LFO resulted in a significant reduction in body weight, waist circumference, and BMI from the baseline, with the degree of the reduction in these three parameters in the LFO group being significantly greater than in the placebo group.

Tominaga et al (2006) reported that the ingestion of 3-fold diluted LFO at a daily dose of $300 \mathrm{mg}$ (equivalent to $100 \mathrm{mg}$ of Flabonoid ${ }^{\mathrm{TM}}$ ) by mildly-obese healthy subjects between 24 and 64 years of age with a BMI between 24 and $30 \mathrm{~kg} / \mathrm{m}^{2}$ for 12 weeks resulted in significant differences with regards to changes in body weight and BMI between the LFO group and the placebo group at week 4,8 , and 12 after administration, in addition to post-ingestion at week 4 [19]. Furthermore, Tominaga et al (2009) also determined the effect of 3-fold diluted LFO intake at a daily dose of $900 \mathrm{mg}$ (equivalent to $300 \mathrm{mg}$ of Flabonoid ${ }^{\mathrm{TM}}$ ) in mildly-obese healthy subjects between 40 and 60 years of age with a BMI between 24 and $30 \mathrm{~kg} / \mathrm{m}^{2}$ for 8 weeks on total body fat and visceral fat together with body weight, BMI, and blood chemistry. Ingestion of LFO resulted in a significant decrease from baseline levels in total body fat mass, visceral fat area, total-cholesterol, and LDL-cholesterol at week 8 , and in body weight and BMI at week 4 and 8. Furthermore, the changes of body weight and that of BMI in LFO group were significantly greater than those in the placebo group [21].

Therefore, the findings reported within this study, as well as from previous clinical studies, indicate that LFO is effective in at least reducing body weight and BMI in mildly-obese adults with or at risk of metabolic syndrome.

LFO intake resulted in significant reduction of the body weight, waist circumference, and 
BMI, although body fat composition did not decrease after ingestion of LFO in this study. The reason for this discrepancy is not clear. However, the changes in fat composition, along with the body weight loss of $2.4 \%$ observed in this study may be too minimal to be accurately assessed by the bioelectrical impedance method [25]. On the other hand, Tominaga et al (2009) [21] concluded that the body weight-reducing effect of LFO was attributable to reduced body fat amount, based on the measurement of body fat composition by dual-energy X-ray absorptiometry (DXA), a reliable and accurate method for assessing body composition [26].

Significant reduction of body weight, waist circumference, and BMI was also observed in placebo group, with $24 \%$ of their subjects not being categorized as having metabolic syndrome at week 12. Although the reason for this outcome is still up to debate, one explanation may be related to the increase of health consciousness within subjects in the placebo group due to their participation in this study, accompanied with their lifestyle change.

Although body fat composition did not decrease after ingestion of LFO in this study, while it has also been reported that waist circumference and BMI are positively associated with abdominal fat volume including visceral adipose tissue $[27,28]$, the present results indicate that the reduction of waist circumference and BMI by LFO could be associated with the reduction of visceral adipose tissue. It is well known that excessive visceral adipose tissue plays a crucial role in triggering the development of metabolic syndrome and its progression [29-31]. With regards to the mechanism by which LFO suppresses visceral fat accumulation, up-regulating genes for beta-oxidation and down-regulation those for fatty acid synthesis have been suggested based on investigations using DNA microarray analysis and subsequent enzymatic analysis with mice and rats treated with LFO [32, 33]. Furthermore, it has also been suggested that LFO enhances fat oxidation in humans, based on the finding that LFO intake increased body trunk surface temperature during cold exposure, decreased respiratory exchange ratio during moderate exercise and during recovery period, and increased oxygen consumption during moderate exercise in healthy humans [34]. For all of these reasons, our observation described above appears to suggest the utility of LFO in the treatment of metabolic syndrome. In fact, at the end of the present study, the percentage of subjects in the LFO group who were not categorized as having metabolic syndrome tended to be greater than those in the placebo group ( $\mathrm{p}=0.077$ ). Accordingly, at the end of this study, the prevalence of subjects who showed improvement in both waist circumference and normal HDL in LFO group was significantly higher than that in the placebo group ( $<<0.05)$, and mean changes from baseline in body weight, waist circumference, BMI, and HDL in LFO group were significantly greater, or tend to be greater than those in the placebo group ( $<<0.01, p<0.001 p<0.01$ or $p=0.075$ respectively); the affirmative effect of LFO on these criteria may mainly contribute to improving metabolic syndrome.

Nevertheless, our study has several limitations. These limitations include a limited number of subjects, a single daily dosing of $300 \mathrm{mg}$, a rather short observation period of 12 weeks, absence of direct determination of visceral adipose tissue amount, and the absence of any effect attributable to LFO on lipid profile and glycemic control. Further studies with a larger number of 
subjects, multiple dosages, and a longer treatment period are necessary to confirm the effects of LFO on metabolic syndrome.

\section{CONCLUSIONS}

The present study suggests that LFO is a promising dietary nutrient for improving metabolic syndrome through its beneficial effect of normalizing body weight, BMI, and possibly the amount of visceral fat and HDL cholesterol.

Abbreviations: licorice flavonoid oil (LFO), body mass index (BMI), National Cholesterol Education Program Adult Treatment panel III (NCEP ATP III), Institutional Review Board (IRB), International Conference on Harmonization-Good Clinical Practice (ICH-GCP)

Conflict of Interest: All authors declare that they have no conflicts of interest.

Author's Contributions: SKP, KN, TU, MH and SJ designed the research; SKP and SJ performed the clinical study and analyzed the data; SKP, KH and HM drafted the manuscript. All authors read and approved the final manuscript.

Acknowledgements: The authors are grateful to Dr. V. Kiran Kumar for managing the clinical studies and providing valuable input.

\section{REFERENCES:}

1. Zimmet P, Magliano D, Matsuzawa Y, Alberti G and Shaw J, The metabolic syndrome: a global public health problem and a new definition. J Atheroscler Thromb 2005; 12:295-300.

2. Kaur J, A comprehensive review on metabolic syndrome. Cardiol Res Pract 2014; 943162, DOI: $10.1155 / 2014 / 943162$.

3. Gregório BM, De Souza DB, de Morais Nascimento FA, Pereira LM, and Fernandes-Santos C, The potential role of antioxidants in metabolic syndrome. Curr Pharm Des 2016; 22: 859-69.

4. Woting A and Blaut M, The intestinal microbiota in metabolic disease. Nutrients 2016; 8, 202; doi:10.3390/nu8040202.

5. Nagao $\mathrm{K}$ and Yanagita T, Functional lipids in metabolic syndrome. J Nutr Sci Vitaminol (Tokyo) 2015; 61 Suppl: S159-161.

6. Haraguchi, H, Yoshida N, Ishikawa H, Tamura Y, Mizutani K and Kinoshita T, Protection of mitochondrial functions against oxidative stresses by isoflavans from Glycyrrhiza galbra. J Pharm Pharmacol 2000; 52:219-223.

7. Rosenblat M, Belinky P, Vaya J, Levy R, Hayek T, Coleman R, Merchav S and Aviram M, Macrophage enrichment with the isoflavan glabridin inhibits NADPH oxidase-induced cell mediated oxidation of low density lipoprotein. A possible role for protein kinase C. J Biol 
Chem 1999; 274:13790-13799.

8. Belinky PA, Aviram M, Mahmood S and Vaya J, Structural aspects of the inhibitory effect of glabridin on LDL oxidation. Free radical Biol Med 1998; 24:1419-1429.

9. Belinky PA, Aviram M, Fuhrman B, Rosenblat M and Vaya J, The antioxidative effects of the isoflavan Glabridin on endogenous constituents of LDL during its oxidation. Atherosclerosis 1998; 137:49-61.

10. Vaya J, Belinky PA and Aviram M, Antioxidant constituents from licorice roots: isolation, structure elucidation and anti oxidative capacity toward LDL oxidation. Free Radical Biol Med 1997; 23:302-313.

11. Fukai T, Marumo A, Kaitou K, Kanda T, Terada S and Nomura T, Anti-Helicobacter pylori flavonoids from licorice extract. Life Sci 2002; 71:1449- 1463.

12. Fukai T, Satoh K, Nomura T and Sakagami H, Antinephritis and radical scavenging activity of prenylflavonoids. Fitoterapia 2003; 74:720-724.

13. Ofir R, Tamir S, Khatib S and Vaya J, Inhibition of serotonin re uptake by licorice constituents. J Mol Neurosci 2003; 20:135-140.

14. Yokota T, Nishio H, Kubota Y and Mizoguchi M, The inhibitory effect of glabridin from licorice extracts on melanogenesis and inflammation. Pigment Cell Res 1998; 11:355-361.

15. Nakagawa K, Kitano M, Kishida H, Nabae K, Kawabe M and Hosoe K, 90-Day repeated-dose toxicity study of licorice flavonoid oil (LFO) in rats. Food and Chem Toxicol 2008; 46:2349-2357.

16. Nakagawa K, Hidaka T, Kitano M, Asakura M, Kamigaito T, Noguchi T et al, Genotoxicity studies on licorice flavonoid oil (LFO). Food Chem Toxicol 2008; 46: 2525-2532.

17. Nakagawa K, Hosoe K, Hodaka T, Nabae K, Kawabe M and Kitano M. Inhibition by licorice flavonoid oil of glutathione S-transferese-positive foci in the medium-term rat hepatocarcinogenesis bioassay. Nutr Res 2010; 30:74-81.

18. Aoki F, Nakagawa K, Kitano M, Ikematsu H, Nakamura K, Yokota S et al, Clinical safety of licorice flavonoid oil (LFO) and pharmacokinetics of glabridin in healthy humans. Journal of the American College of Nutrition 2007; 26:209-218.

19. Tominaga Y, Mae T, Kitano M, Sakamoto Y, Ikematsu H and Nakagawa K, Licorice flavonoid oil effects body weight loss by reduction of body fat mass in overweight subjects. J Health Sci 2006; 52:672-683.

20. Nakagawa K, Kishida H, Arai N, Nishiyama T and Mae T, Licorice flavonoids suppress abdominal fat accumulation and increase in blood glucose level in obese diabetic KK-A(y) mice. Biol Pharm Bull 2004; 27:1775-1778.

21. Tominaga Y, Nakagawa K, Mae T, Kitano M, Yokota S, Arai T et al, Licorice flavonoid oil reduces total body fat and visceral fat in overweight subjects, A randomized, double-blind, placebo-controlled study. Obes Res Clin Pract 2009; 3:169-178.

22. Wasir JS, Misra A, Vikram NK, Pandey RM and Gupta R. Comparison of Definitions of the 
Metabolic Syndrome in Adult Asian Indians. J Assoc Physicians India 2008;56:158-164.

23. Julious SA, Sample size of 12 per group rule of thumb for a pilot study. Pharm Stat 2005; 4:287-291.

24. Hertzog MA, Considerations in determining sample size for pilot studies. Res Nurs Health 2008; 31:180-91.

25. Verdich C, Barbe P, Petersen M, Grau K, Ward L, Macdonald I et al, Changes in body composition during weight loss in obese subjects in the NUGENOB study: comparison of bioelectrical impedance vs. dual-energy X-ray absorptiometry. Diabetes Metab 2011; 37:222-229.

26. Glickman SG, Marn CS, Supiano MA and Dengel DR, Validity and reliability of dual-energy X-ray absorptiometry for the assessment of abdominal adiposity. J Appl Physiol (1985) 2004; 97:509-514.

27. Camhi SM, Bray GA, Bouchard C, Greenway FL, Johnson WD, Newton RL et al, The relationship of waist circumference and BMI to visceral, subcutaneous, and total body fat: sex and race differences. Obesity (Silver Spring) 2011; 19:402-408.

28. Berg RM, Wallaschofski H, Nauck M Rettig R, Markus MR, Laqua R et al, Positive Association between adipose tissue and bone stiffness. Calcif Tissue Int 2015; 97:40-49.

29. Guize L, Panniera B, Thomasa F, Bean K, Jégo B and Benetos A, Recent advances in metabolic syndrome and cardiovascular disease. Arch Cardiovasc Dis 2008; 101:577-583.

30. Huffman DM and Barzilai1 N, Role of visceral adipose tissue in aging. Biochim Biophys Acta 2009; 1790:1117-1123.

31. Vissers D, Hens W, Taeymans J, Baeyens JP, Poortmans J and Van Gaal L, The Effect of exercise on visceral adipose tissue in overweight adults: A systematic review and meta-analysis. PLoS One 2013; 8, e56415, DOI: 10.1371/journal.pone.0056415.

32. Aoki F, Honda S, Kishida H, Kitano M, Arai N, Tanaka H et al, Suppression by licorice flavonoids of abdominal fat accumulation and body weight gain in high-fat diet-induced obace C57BL/6J mice. Biosci Biotechnol Biochem 2007; 71:206-214.

33. Kamisoyama H, Honda $\mathrm{K}$, Tominaga $\mathrm{Y}$, Yokota $\mathrm{S}$ and Hasegawa $\mathrm{S}$, Investigation of the anti-obesity action of licorice flavonoid oil in diet-induced obese rats. Biosci Biotechnol Biochem 2008; 72:80469-1-7.

34. Mori N, Nakanishi S, Shiomi S, Kiyokawa S, Kakimoto S, Nakagawa K et al, Enhancement of fat oxidation by licorice flavonoid oil in healthy humans during light exercise. J Nutr Sci Vitaminol 2015; 61:406-416. 